\title{
Visual acuity after acute primary angle closure and considerations for primary lens extraction
}

\author{
G S W Tan, S-T Hoh, R Husain, G Gazzard, F T S Oen, S K L Seah, T Aung
}

Br J Ophthalmol 2006;90:14-16. doi: 10.1136/bjo.2005.077115

\begin{abstract}
Background/aim: Primary lens extraction has been advocated for acute primary angle closure (APAC), but it is not known if this is warranted in all cases. The aim of this study was to investigate the visual acuity (VA) of APAC eyes shortly after resolution of the acute episode in order to assess the appropriateness of performing such surgery in this condition.

Methods: This was a prospective observational case series. As part of a randomised controlled trial comparing phacoemulsification and laser iridotomy, 135 consecutive APAC subjects over a 2 year period underwent subjective refraction and measurement of Snellen VA once the acute episode had resolved with reduction of intraocular pressure (IOP) and improved corneal clarity.

Results: Subjects were predominantly Chinese (95.6\%) and female $(79.3 \%)$, with a mean age of 63.6 (SD 9.6) years. When assessed 1.7 (2.7) days after presentation, the majority of APAC cases (50.4\%) had good VA (6/12 or better), with more than a quarter of cases having VA of $6 /$ 7.5 or better. Poor VA was associated with duration of symptoms $(p=0.04, O R=4.1,95 \% \mathrm{Cl} 1.1$ to 15.7$)$ and time taken to resolution of APAC $(p=0.04, O R=2.2,95 \% \mathrm{Cl}$ 1.02 to 4.6$)$, but not with sex $(p=0.31)$, age $(p=0.26)$, duration from presentation to measurement of visual acuity $(p=0.53)$, or presenting IOP $(p=0.73)$.

Conclusion: Within days after APAC, more than half of APAC affected eyes had good VA (6/12 or better). The role of lens extraction in the management of APAC warrants further debate, especially for eyes with good VA.
\end{abstract}

\footnotetext{
A cute primary angle closure (APAC) is a potentially blinding ocular condition, with high incidence especially in east Asia. ${ }^{1}$ Laser peripheral iridotomy (LPI), performed to eliminate pupil block, is the current standard therapy for APAC. However, after LPI, many Asian eyes with APAC still develop uncontrolled intraocular pressure (IOP) requiring further treatment. ${ }^{2}$ Significant ocular morbidity and blindness have also been reported in APAC eyes several years after LPI. ${ }^{3}$

As the lens is thought to play a major part in the pathogenesis of $\mathrm{APAC}^{45}$ primary lens extraction has been advocated as an alternative to LPI in the management of APAC. ${ }^{67}$ This has prompted randomised controlled trials comparing primary phacoemulsification with laser iridotomy to commence in Hong Kong (Lam DSC, personal communication) and in Singapore. The aim of this study was to investigate the visual acuity (VA) of APAC eyes shortly after resolution of the acute episode in order to assess the appropriateness of performing primary phacoemulsification in such patients.
}

\section{METHODS}

As part of a randomised controlled trial comparing LPI and primary phacoemulsification in the treatment of APAC, consecutive subjects attending our centre over 2 years underwent a standardised protocol of management. Written informed consent was obtained from all subjects and the study had the approval of the ethics committee of the Singapore National Eye Centre, and was performed according to the tenets of the Declaration of Helsinki. The following criteria were used to define cases of APAC:

- Presence of at least two of the following symptoms: ocular or periocular pain, nausea and/or vomiting, an antecedent history of intermittent blurring of vision with haloes;

- Presenting IOP of more than $28 \mathrm{~mm} \mathrm{Hg}$ (as measured by Goldmann applanation tonometry) and the presence of at least three of the following signs: conjunctival injection, corneal epithelial oedema, mid-dilated unreactive pupil, and shallow anterior chamber.

Upon presentation, APAC cases were initially managed by drugs in order to reduce IOP. This consisted of intravenous acetazolamide (followed by oral acetazolamide), topical pilocarpine, timolol, and betamethasone or prednisolone acetate. If the IOP did not reduce with this standard treatment, other medications such as intravenous mannitol, oral glycerol, topical latanoprost, or brimonidine were added. Once the acute episode had resolved with reduction of IOP below $21 \mathrm{~mm} \mathrm{Hg}$ with improved corneal clarity, subjects underwent subjective refraction and measurement of Snellen VA, before being considered for randomisation to either of the two treatment arms.

\section{RESULTS}

The demographic characteristics and presenting clinical features of the first 135 consecutive eligible subjects are summarised in table 1. Subjects were predominantly Chinese $(95.6 \%)$ and female $(79.3 \%)$, with a mean age of 63.6 (SD 9.6) years. Best corrected VA of study subjects was assessed 1.7 (2.7) days after presentation. Interestingly, we found that the majority of APAC cases (50.4\%) had good VA (6/12 or better), with $27 \%$ of cases having VA of $6 / 7.5$ or better (table 2).

Poorer VA was associated with duration of symptoms $(\mathrm{p}=0.04, \mathrm{OR}=4.1,95 \%$ CI 1.1 to 15.7$)$ and time taken to resolution of APAC $(\mathrm{p}=0.04, \mathrm{OR}=2.2,95 \%$ CI 1.02 to 4.6$)$, but not with sex $(p=0.31)$, age $(p=0.26)$, duration from presentation to measurement of visual acuity $(p=0.53)$, or presenting IOP $(p=0.73)$. Eight subjects had documented co-morbid diseases which caused poor visual acuity: these were retinitis pigmentosa (two subjects), epiretinal membrane (two subjects), central retinal vein occlusion (one

Abbreviations: APAC, acute primary angle closure; IOP, intraocular pressure; LPI, laser peripheral iridotomy; VA, visual acuity 
Table 1 Demographic characteristics and presenting clinical features of patients with acute primary angle closure in this study

\begin{tabular}{ll}
\hline $\begin{array}{l}\text { Age (years) } \\
\text { Mean }\end{array}$ & $63.6($ SD 9.6) \\
Range & $42-89$ \\
Sex & $28(20.7 \%)$ \\
Male & $107(79.3 \%)$ \\
Female & $129(95.6 \%)$ \\
Race & $4(3.0 \%)$ \\
Chinese & $2(1.5 \%)$ \\
Malay & \\
Indian & $65(48.1 \%)$ \\
Laterality & $70(51.9 \%)$ \\
$\quad$ Right & $58.6(12.8$ (range 6-80) \\
Left & $2.8($ SD 3.2) (range 1 hour-21 days) \\
Presenting IOP (mm Hg) & \\
Duration of symptoms (days) & \\
\hline
\end{tabular}

subject), macular hole (one subject), Fuch's endothelial dystrophy (one subject), and age related macular degeneration (one subject).

\section{DISCUSSION}

Our results raise several management and ethical questions regarding the role of primary lens extraction in the management of APAC. As the majority of eyes in this study had good VA of $6 / 12$ or better within days of presentation, primary phacoemulsification may not be justified for all cases of APAC. For the trial we are conducting, our institutional review board only permitted subjects with VA of $6 / 15$ or worse to undergo randomisation. The relatively good VA found in most of the APAC subjects so far has thus limited the recruitment of patients for our trial. Should eyes with better acuities and clearer lenses also be considered for primary phacoemulsification? Apart from eliminating pupil block, a possible benefit of early lens removal is widening and deepening of the angle, ${ }^{8}$ which may potentially prevent future IOP rise and the development of chronic glaucoma with optic nerve damage. This consideration may be especially valuable in settings where long term follow up of patients may be problematic. These potential benefits must be weighed against the loss of accommodation after lens extraction and the risks of surgery such as endophthalmitis, suprachoroidal haemorrhage, and retinal detachment.

Cataract surgery in eyes with previous APAC is technically difficult and significant intraoperative and postoperative complications have been reported for APAC cases undergoing phacoemulsification. ${ }^{6}$ The anterior chamber is usually shallow, the lens often bulky, and the pupil is frequently small from miotic treatment or ischaemic iris atrophy. Iris ischaemia from the acute episode may render the iris floppy, which together with a small pupil, may make the iris more susceptible to trauma during surgery. In addition, poor cornea clarity makes the surgical outcome very much dependent on the skill and experience of the surgeon. Corneal decompensation is another potential long term complication. The corneal endothelium, already at risk of damage from the acute episode, ${ }^{9}$ may undergo further injury from the phacoemulsification surgery leading to subsequent decompensation.

Important issues related to primary lens extraction for APAC remain unresolved. The first is the optimum timing of lens extraction after resolution of the APAC episode. In our randomised controlled trial, primary phacoemulsification is performed in eligible cases within days of resolution of the acute episode. It is not known if there should be a delay of a few weeks to reduce intraocular inflammation and improve
Table 2 Best corrected visual acuity in affected eyes of individuals with acute primary angle closure after resolution of the acute event

\begin{tabular}{lrl}
\hline Visual acuity & No & $\%$ \\
\hline$\geqslant 6 / 7.5$ & 37 & 27.4 \\
$6 / 9-6 / 12$ & 31 & 23.0 \\
$6 / 15-6 / 24$ & 34 & 25.2 \\
$6 / 30-6 / 48$ & 8 & 5.9 \\
$<6 / 60$ & 25 & 18.5 \\
\hline
\end{tabular}

corneal clarity. Should a laser iridotomy be performed first during this interval? In view of the demands and dangers of primary lens extraction, perhaps it may be safer and more appropriate to perform LPI first in all cases, regardless of the extent of visually significant cataract. Patients could then be followed up and if there is significant cataract, lens extraction may be considered a few weeks to months after resolution of the acute episode. Another consideration for surgical indication and outcome is the significance of non-lens factors such as the extent of synechiael angle closure and the degree of optic nerve cupping. Significant synechiae or advanced optic disc cupping may suggest underlying chronic angle closure glaucoma for which lens extraction alone may not be sufficient for long term IOP control. ${ }^{10}{ }^{11}$ In such cases, lens extraction may need to be combined with trabeculectomy ${ }^{12}$ or goniosynechialysis. ${ }^{13}$

Our study had several limitations. The study population was Asian (predominantly Chinese), and it is not known if the results could be applied to other racial groups. We did not assess the optic disc in a standardised manner in order to ensure that reduced visual acuity was not the result of glaucomatous optic nerve head damage. There was lack of longer term follow up data on IOP, peripheral anterior synechiae, and cataract progression which may provide information on the ideal time for lens extraction following APAC. Finally, we excluded those who did not respond to drugs and our analysis and results are thus limited to a selected subpopulation of medically responsive APAC subjects. Cases in which IOP control following an APAC attack is difficult may be more likely to have a phacomorphic component and therefore worse VA.

In summary, we believe that the role of lens extraction in the management of APAC is a subject that merits further research and discussion. Primary surgery may not always be justifiable given that the majority of cases have good visual acuity after medical resolution of the acute event. More randomised controlled trials are essential but such studies will require careful planning and stratification of patients in order to help resolve the issues at hand and yet avoid exposing patients to unjustifiable risks.

\section{Authors' affiliations \\ G S W Tan, Sek-Tien Hoh, R Husain, G Gazzard, F T S Oen, S K L Seah, \\ T Aung, Singapore National Eye Centre, Singapore \\ R Husain, G Gazzard, Institute of Ophthalmology, London, UK \\ T Aung, National University of Singapore, Singapore}

This study was supported by grants from the Singapore Eye Research Institute and Singhealth Group, Singapore.

Commercial interest: The authors have no financial or other conflict of interest with regard to the paper.

Correspondence to: Dr Tin Aung, Glaucoma Department, Singapore National Eye Centre, 11 Third Hospital Avenue, Singapore 168751: tin11@pacific.net.sg

Accepted for publication 5 September 2005 


\section{REFERENCES}

1 Seah SK, Foster PJ, Chew PT, et al. Incidence of acute primary angle-closure glaucoma in Singapore. An island-wide survey. Arch Ophthalmol 1997; 115:1436-40.

2 Aung T, Ang LP, Chan SP, et al. Acute primary angle-closure: long-term intraocular pressure outcome in Asian eyes. Am J Ophthalmol $2001 ; 131: 7-12$.

3 Aung T, Friedman DS, Chew PTK, et al. Long-term outcomes in Asians after acute primary angle-closure. Ophthalmology 2004;111:1464-9.

4 Hung PT, Chou LH. Provocation and mechanism of angle-closure glaucoma after iridectomy. Arch Ophthalmol 1979;97:1862-4.

5 Ritch R, Lowe RF. Angle closure glaucoma: Mechanisms and epidemiology. In Ritch R, Shields MB, Krupin T, eds. The glaucomas. St Louis: CV Mosby, 1996 chap 37, 801-19.

6 Jacobi PC, Dietlein TS, Luke C, et al. Primary phacoemulsification and intraocular lens implantation for acute angle-closure glaucoma Ophthalmology 2002;109:1597-603

7 Ming ZZ, Lim AS, Yin WT. A pilot study of lens extraction in the management of acute primary angle-closure glaucoma. Am J Ophthalmol 2003; 135:534-6.
8 Hayashi $\mathrm{K}$, Hayashi $\mathrm{H}$, Nakao $\mathrm{F}$, et al. Changes in anterior chamber angle width and depth after intraocular lens implantation in eyes with glaucoma. Ophthalmology 2000; 107:698-703.

9 Sihota R, Lakshmaiah NC, Titiyal JS, et al. Corneal endothelial status in the subtypes of primary angle closure glaucoma. Clin Experiment Ophthalmol 2003;31:492-5.

10 Nolan WP, Foster PJ, Devereux JG, et al. YAG laser iridotomy treatment for primary angle closure in east Asian eyes. $\mathrm{Br} J$ Ophthalmol 2000:84:1255-9.

11 Alsagoff Z, Aung T, Ang LPK, et al. Long-term clinical course of primary angle-closure glaucoma in an Asian population. Ophthalmology 2000; 107:2300-4.

12 Tow SLC, Aung T, Oen FTS, et al. Combined phacoemulsification, intraocular lens implantation and trabeculectomy in Asian patients with chronic angle closure glaucoma. Int Ophthalmol $2001 ; 24: 283-9$.

13 Teekhasaenee C, Ritch R. Combined phacoemulsification and goniosynechialysis for uncontrolled chronic angle-closure glaucoma after acute angle-closure glaucoma. Ophthalmology 1999;106:669-75

\section{bmjupdates+}

bmjupdates+ is a unique and free alerting service, designed to keep you up to date with the medical literature that is truly important to your practice.

bmjupdates+ will alert you to important new research and will provide you with the best new evidence concerning important advances in health care, tailored to your medical interests and time demands.

\section{Where does the information come from?}

bmiupdates+ applies an expert critical appraisal filter to over 100 top medical journals A panel of over 2000 physicians find the few 'must read' studies for each area of clinical interest

Sign up to receive your tailored email alerts, searching access and more...

www.bmjupdates.com 